\title{
Longitudinal changes in body composition during ipilimumab treatment for metastatic melanoma
}

\author{
L. Daly ${ }^{1}$, A. O’Reilly ${ }^{2}$, D.G. Power ${ }^{3}$, S. Cushen ${ }^{1}$, D. Woodlock ${ }^{4}$ and A. Ryan ${ }^{1}$ \\ ${ }^{1}$ School of Food and Nutritional Sciences, University College Cork, Ireland, ${ }^{2}$ Department of Medical Oncology \& \\ ${ }^{4}$ Radiology, University Hospital Galway, Ireland and ${ }^{3}$ Department of Medical Oncology, Mercy \& Cork University \\ Hospitals, Cork, Ireland
}

Ipilimumab is a novel monoclonal antibody used in the treated of metastatic melanoma. Sarcopenia has recently emerged as an important predictor chemotherapeutic drug efficacy and toxicity ${ }^{(1,2)}$ and as well as poor performance status and shortened survival in cancer patients ${ }^{(1)}$. Sarcopenia can develop rapidly after a diagnosis of cancer, particularly in the metastatic setting. To-date no studies have examined the prevalence of sarcopenia in metastatic melanoma or the impact of ipilimumab on muscle mass and clinical outcomes.

The aim of this study was to examine the prevalence of sarcopenia in metastatic melanoma and to examine the impact of ipilimumab on longitudinal changes in body composition. Patients with metastatic melanoma, treated with ipilimumab between 2009-2015 at two university teaching hospitals were included. Body composition was assessed by CT scan ${ }^{(2)}$ at the 3rd lumbar vertebra using OsiriX $^{\circledR}$ software (Pixmeo, Geneva, Switzerland) at baseline and after 4 cycles of Ipilmumab (12 weeks) and included measurement of skeletal muscle, visceral and subcutaneous adipose tissue. Cut off points for sarcopenia were set at $55.4 \mathrm{~cm}^{2} / \mathrm{m}^{2}$ for men and $38.9 \mathrm{~cm}^{2} / \mathrm{m}^{2}$ for women ${ }^{(2)}$. Estimates of whole body fat free mass (FFM) were calculated using published regression equations ${ }^{(2)}$.

Seventy-four patients were treated with Ipilimumab, however only 42 patients had both baseline and 3-month imaging available for analysis. There were 24 men $(57 \%)$ and 18 women. The mean age was $53( \pm 17)$ years and the mean BMI was $28.15 \pm 6.5 \mathrm{~kg} / \mathrm{m}^{2} .71 \%$ of patients were considered to be overweight or obese according to WHO standards. Sarcopenia was present at baseline in $31 \%$ of patients and it was prevalent across all BMI categories. Skeletal muscle area (SMA) was significantly reduced from baseline to follow up $\left(150.1 \pm 38.8\right.$ vs $\left.145.4 \pm 39.7 \mathrm{~cm}^{2}, p=0.047\right)$. Mean SMA change was $4.74 \pm 14.9 \mathrm{~cm}^{2} .38 \%$ of patients experienced a meaningful muscle loss of $>6 \mathrm{~cm}^{2}$ between the baseline and follow up scan (14.3\% experienced meaningful muscle gain). This cut point was chosen, as it is equivalent to $1 \mathrm{~kg}$ of skeletal muscle and is associated with physical function ${ }^{(3)}$. Fat free mass significantly decreased from $51.09 \mathrm{~kg}( \pm 11.65)$ to $49.67 \mathrm{~kg}( \pm 11.18)(\mathrm{p}=0.04)$ and muscle density was also significantly reduced (mean loss of 4.15 mean $\mathrm{HU})(\mathrm{P}=$ 0.001). The prevalence of sarcopenia thereby increased significantly from $31 \%(\mathrm{n}=13)$ at baseline to $52.4 \%(\mathrm{n}=22)$ by completion of Ipilimumab $(p=0.004)$. Adipose tissue index $\left(\mathrm{cm}^{2} / \mathrm{m}^{2}\right)$ and fat mass $(\mathrm{kg})$ were also significantly reduced $(\mathrm{p}=0.01, \mathrm{p}=0.026$ respectively). $45 \%$ of patients experienced a meaningful adipose tissue loss of $>14 \cdot 7 \mathrm{~cm}^{2(3)}$, with a mean loss of $28 \cdot 6 \pm 70 \cdot 15 \mathrm{~cm}^{2}$.

Fig. 1. Male, 47 years old. SMA at L3 before (left) and after (right) treatment. Images taken 3 months apart. $26 \cdot 8 \mathrm{~cm}^{2}$ loss of muscle. SMI reduced from $62 \mathrm{~cm}^{2} / \mathrm{m}^{2}$ (non-sarcopenic) to $54.7 \mathrm{~cm}^{2} / \mathrm{m}^{2}$ (sarcopenic)
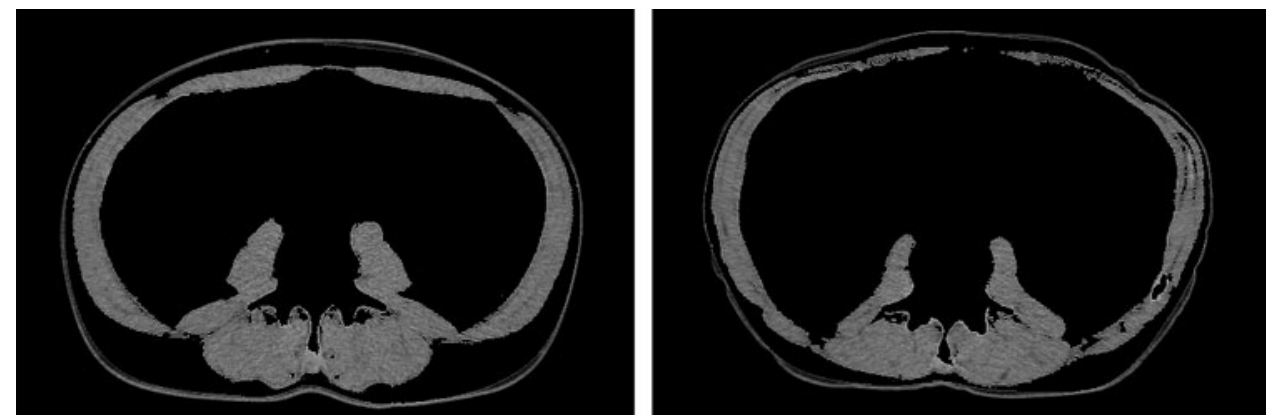

While this study was limited in terms of sample size, we observed significant and rapid loss of muscle within this patient group over a short time frame. These changes in muscle mass cannot be explained by the age related loss of muscle since measurements were taken within 5 months. The reduction in muscle mass may relate to fatigue experienced by patients during treatment resulting in reduced physical activity. $52.4 \%$ of patients experienced fatigue (any grade), while $19 \%$ reported experiencing grade 3 or 4 fatigue.

In conclusion metastatic melanoma patients treated with Ipilimumab experience significant and rapid loss of muscle mass during treatment with Ipilimumab. This muscle loss is masked by excessive adiposity. Patients should be encouraged toward isometric exercise and nutritional intervention to maximise protein intake in the hope of retaining muscle mass.

1. Prado CM, Lieffers JR, McCargar LJ et al. (2008) Lancet Onco 9, 629-635.

2. Mourtzakis M, Prado CM, Lieffer JR et al. (2008) Appl Physiol, Nutr Metab 33, 997-1006.

3. Prado CM, Sawyer MB, Gosh S et al. (2013) Am J Clin Nutr 98, 1012-1019. 